\title{
Low Cost, Continuously Tunable, Millimeter-wave Photonic LO Generation using Optical Phase Modulation and DWDM Filters
}

\author{
P. Shen, J. James, N. J. Gomes, P. G. Huggard and B. N. Ellison
}

\begin{abstract}
We report on a low cost technique for the photonic generation of wideband continuously tunable millimeter-wave local oscillator signals. It is based on side band filtering using an optical phase modulator and thin film DWDM filters. The generated millimeter-wave signal exhibits low phase noise, and its frequency covers the $W$ and F-bands, from $75 \mathrm{GHz}$ to $140 \mathrm{GHz}$.
\end{abstract}

Index Terms-Thin film filter, photonic Local Oscillator, optical phase modulator, millimeter-wave

\section{INTRODUCTION}

$\mathrm{M}$ illimeter-wave (MMW) over fiber systems are considered to be promising for future broadband wireless access services due to the spectral congestion and the transmission bandwidth limitations at microwave frequencies [1]. They also find applications in time/phase reference distribution systems, such as those in phased array or radar systems. Owing to the difficulty in directly modulating lasers at MMW frequencies, the signals required are often generated by means of photonics.

Among MMW generation schemes, external modulation is very attractive due to its capability of producing high purity microwave and MMW signals, as well as its relatively simple implementation. Frequency doubling or quadrupling can be achieved by utilizing the nonlinearity of an optical modulator, thereby reducing the modulation bandwidth requirement on the optical modulators. Both intensity [2-4], and phase [5-7] modulators can be used in such a system. When the frequency moves into the MMW band, one can adopt optical filters to select the wanted sidebands to enhance the MMW generation. Different filtering configurations, such as cascading the phase modulator with a polarizer [5], or by using an optical loop mirror, a Faraday mirror, a narrowband Fiber Bragg Grating (FBG) $[6,7]$, an Arrayed Waveguide Grating (AWG) or a Fabry Perot filter $[\underline{8,9]}$ have been implemented previously.

One can use two narrow band filters, such as FBGs, or a demultiplexing filter, such as an AWG, to select the wanted sidebands, and then combine them together to 'compose' a

Manuscript received March 15, 2008. This work was funded by the UK Engineering and Physical Sciences Research Council (EPSRC) through the COMCORD project.

P. Shen, J. James and N.J. Gomes are with the Broadband and Wireless Communications Group, Department of Electronics, University of Kent, Canterbury, CT2 7NT, UK (e-mail: p.shen@kent.ac.uk).

P. G. Huggard and B. N. Ellison are with the Space Science and Technology Department, STFC, Rutherford Appleton Laboratory, Chilton, Didcot OX11 0QX, UK photonic MMW signal, as reported in [3]. In this approach, the two selected optical sidebands are transported in different fibers before they are combined. Polarization control of the individual sidebands is necessary to match the polarization states of the two selected sidebands, thereby maximizing the efficiency of the MMW generation. Furthermore, the path lengths in the differential paths may need to be stabilized, as the phase of the generated MMW signal depends directly on the phase difference between the two optical sidebands.

Therefore, techniques which keep the selected sidebands in the same optical path are very attractive for the removal of the aforementioned large phase excursions and for reducing the need for polarization control between the selected sidebands. In [7], this is achieved by employing 7 narrow band FBGs to remove the unwanted sidebands. Nevertheless when the MMW frequency is tuned, the FBGs need to be adjusted accordingly. In [8], where a FP filter is used, the tuning range is limited to a few hundreds of $\mathrm{MHz}$ due to the requirement of high Finesse in the FP filters in order to suppress the unwanted sidebands.

In this letter, a photonic local oscillator (LO) based on sideband filtering of a phase modulated signal is presented. Using the phase modulator offers the advantage of reducing the DC-bias circuitry, as a drift in the bias point of an intensity modulator results in the output optical spectrum changing. Here, we adopt a novel configuration utilizing two cascaded thin film filters to provide a widely tunable MMW photonic LO source. This much simpler configuration possesses a common optical path and we have successfully demonstrated a wide tuning range without the need to adjust the optical filter.

\section{PRINCIPLE AND EXPERIMENTAL SETUP}

When an optical phase modulator is driven by an RF drive signal, its optical output waveform is given by

$$
E_{o}(t)=\hat{E}_{r} e^{-j w_{r} t} e^{-j \beta \sin \left(\omega_{m} t\right)}=\hat{E}_{r} \sum_{n=-\infty}^{\infty}\left[J_{n}(\beta) e^{-j w_{r} t-j n \omega_{m} t}\right]
$$

where $\hat{E}_{r}$ is the optical field amplitude and $\beta$ is the modulation index. $\omega_{r}$ and $\omega_{m}$ are the angular frequencies of the optical carrier and of the electrical drive signal, respectively. The amplitude of the $n^{\text {th }}$ optical sideband $\hat{E}_{r} J_{n}(\beta) e^{-j w_{r} t-j n \omega_{m} t}$ is determined by Bessel functions of the first kind, $J_{n}(\beta)$. Generally speaking, increasing the modulation index increases the power transferred to the higher order sidebands. By adjusting the power level of the RF drive signal, the modulation index can be set such that the optical power in the desired optical sidebands is maximized. 


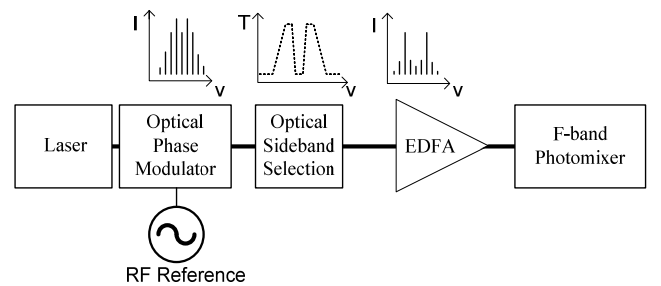

Fig. 1 Use of phase modulator and sideband filtering to produce MMW signals. After filtering the amplified lightwave beat in a photomxier.

A phase modulated optical lightwave has constant intensity. The amplitude and phase relationship between the optical components causes the beat signals produced by those components to cancel, such that no RF signal is detected by a photodiode. Optical filters can be employed to destroy such a relationship. This can be done by selecting the optical sidebands with a desired frequency separation, i.e. the MMW frequency as shown in Fig. 1.

We propose to use an optical filter that has two passbands that are separated by the desired MMW frequency. Such a filter can be fabricated by cascading a bandpass filter with a bandstop filter. The two filter characteristics have the same central wavelength, but different bandwidths. This approach is illustrated in Fig. 2. Two low cost Dense-Wavelength-Division Multiplexing (DWDM) add/drop thin film filters, with 100 and $200 \mathrm{GHz}$ channel separations, are used. The 200 (100) GHz filter is configured in a bandpass (bandstop) mode. The selected sidebands are transmitted through the $200 \mathrm{GHz}$ filter and then reflected by the $100 \mathrm{GHz}$ filter. In this way, the lightwaves of the desired sidebands are always transmitted along the same optical path, therefore eliminating any differential path length changes and any associated MMW phase drift. Furthermore, as both DWDM filters have a polarization mode dispersion of less than $100 \mathrm{fs}$, the polarization misalignment between the two selected sidebands is negligible, with a MMW power penalty below $0.1 \mathrm{~dB}$, so no polarization control is needed.

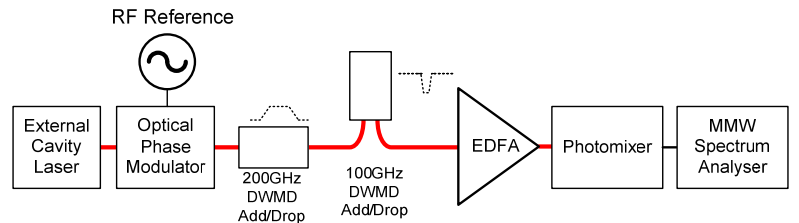

Fig.2 Experimental Configuration: connecting to through port of the $200 \mathrm{GHz}$ DWDM add/drop creates a bandpass filter whereas using the $100 \mathrm{GHz}$ DWMD add/drop in reflection forms a bandstop filter.

To complete the experimental setup, a tunable external cavity laser and an optical phase modulator with a $40 \mathrm{GHz}$ RF bandwidth are used for the initial optical sideband generation. During the experiment, the laser wavelength is set to be at the center of the filter characteristics. It is found that once the wavelength is selected, the stability of the filters and the laser are sufficient so that no further adjustment is required. An Erbium Doped Fiber Amplifier (EDFA) is used to boost the power of the selected optical sidebands before the light is fed into a millimeter wave photomixer.

\section{EXPERIMENTAL RESULTS}

The cascading of the bandpass and bandstop filters yields a spectral transmittance that contains two transmission peaks, as shown in Fig. 3. Both peaks have a passband width of $0.3 \mathrm{~nm}$. We propose to use these dual transmission peaks to select the required sidebands simultaneously. Fig. 4 shows a typical optical spectrum of the phase modulated signal at the output of the filters; in this case the modulator was driven at $25 \mathrm{GHz}$.

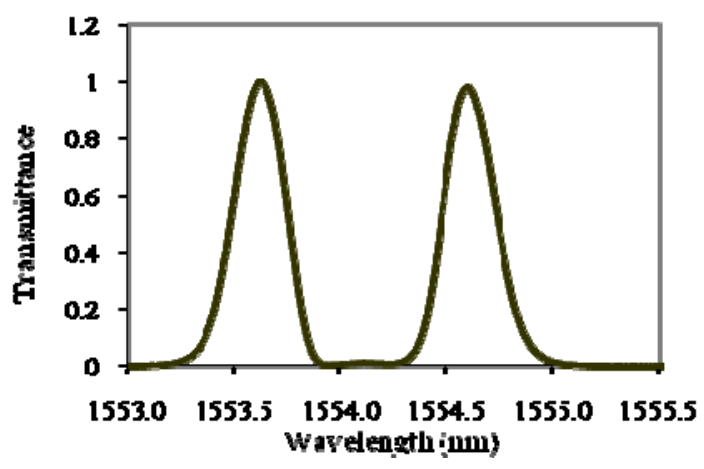

Fig. 3 Spectral transmission of the cascaded DWDM filters.

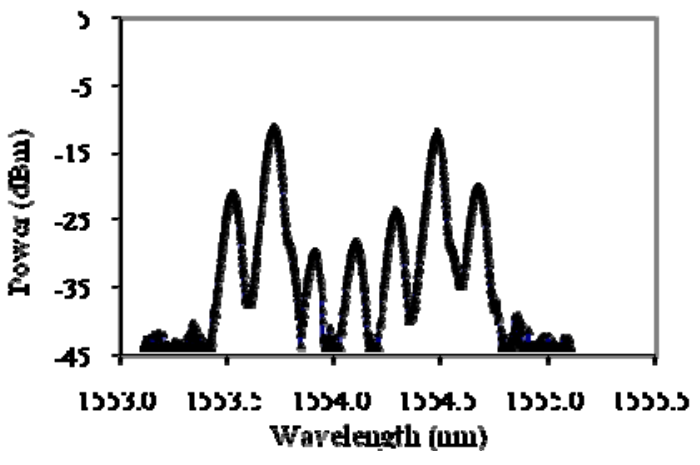

Fig. 4 Typical optical spectrum of the sideband filtering output. $\mathrm{RF}$ modulation at $25 \mathrm{GHz}$

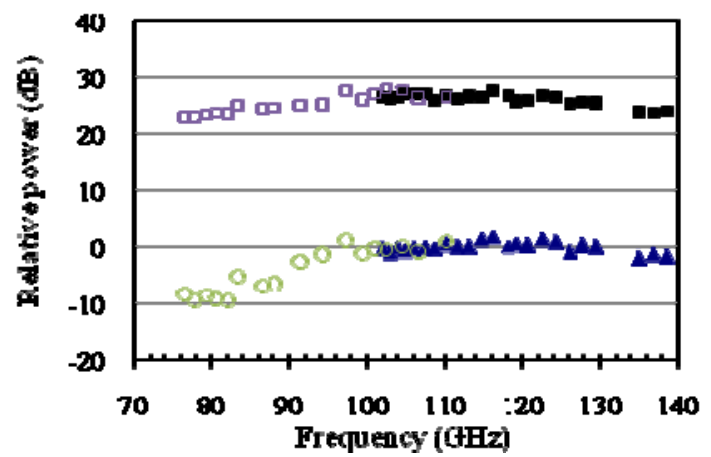

Fig. 5 Relative power of the generated MMW signal after calibration for the photomixer response.

$\square$ W-band photomixer, with EDFA; - F-band photomixer, with EDFA $\circ \mathrm{W}$-band photomixer, without EDFA; $\mathbf{\Delta}$ F-band photomixer, without EDFA

As the dual transmission peaks are relatively wide, the system is expected to offer continuous tunability over tens of GHz. This has been demonstrated in Fig. 5, which shows the relative MMW power received with the RF drive frequency varied to tune the generated signal across the $\mathrm{W}$ - and F-bands. With the same modulation depth for the phase modulated signal, the generated MMW signal exhibits a power flatness to within about $\pm 2 \mathrm{~dB}$ from $92 \mathrm{GHz}$ to $140 \mathrm{GHz}$. At frequencies lower than $92 \mathrm{GHz}$, the MMW power drops by up to $10 \mathrm{~dB}$. This is mainly due to the rolloff of the optical filters. When the 
filtered optical signal is amplified by an EDFA, the detected MMW power has lower frequency dependence $(5 \mathrm{~dB})$ due to the saturation of the EDFA output power.

The phase noise of the generated MMW signal was also examined. Fig. 6 shows typical phase noise performance, in this case of a MMW signal generated at $106.1 \mathrm{GHz}$. The inserted dashed line represents the phase noise of the corresponding $26.525 \mathrm{GHz}$ drive signal. In the region close to the carrier, the phase noise has increased by $12 \mathrm{~dB}$ compared to that of the drive signal. This increase is purely due to the $4 \times$ multiplication factor. This illustrates that the increase in phase noise depends only on the order of the harmonics selected for heterodyning and not from additional system noise. In Fig. 6, the MMW signal phase noise is limited by the noise floor of the measurement system above an offset of $100 \mathrm{kHz}$.

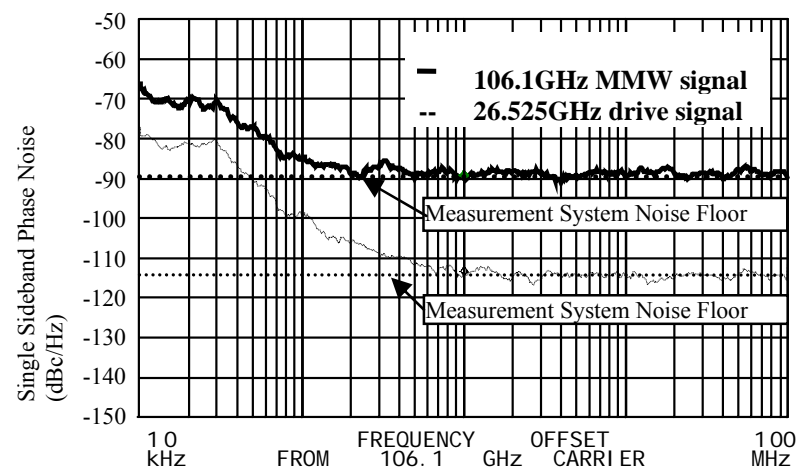

Fig. 6 Phase noise of a typical MMW signal at $106.1 \mathrm{GHz}$ and of the corresponding drive signal. Above an offset of $100 \mathrm{kHz}$, the phase noise of the MMW signal is limited by the measurement system noise floor.

Unsuppressed optical sidebands will cause the output to contain RF signals at other harmonics of the drive frequency. However, this is not a problem in most applications as the photonic LO signal will be filtered by filters and/or narrow band MMW amplifiers, or be used as a reference for a narrowband phase lock loop before being fed into any subsequent system.

Of more concern, is the fact that weaker pairs of unsuppressed sidebands will contribute to the desired MMW signal. Due to the chromatic dispersion of single mode fiber $[10]$, these unsuppressed optical sidebands can lead to additional power variation when the signal is being distributed through a long fiber link. This is a particular worry in this low cost approach, since the unsuppressed optical sideband powers are relatively high. A study of the power penalties has been made by a simulation. Fig. 7 shows the calculated power penalties vs. fiber length for MMW signals at 75, 100 and 140 $\mathrm{GHz}$. The power variations are 3.0, 1.7 and $1.2 \mathrm{~dB}$, respectively The lower frequency suffers greater fading because of worse suppression of the $3^{\text {rd }}$ order optical sidebands.

It should be stressed that the results in Fig. 7 assume the simplest setup, with no adjustments made to modulation depth or filter configurations. The power penalties can be reduced using chromatic dispersion compensation schemes, or by optimizing the optical spectrum by enhancing the selected sidebands. The latter can be done by adjusting the modulation depth, or by using filters with different bandwidth. The simulation shows that the power variations can be reduced to $1.9 \mathrm{~dB}$ at $75 \mathrm{GHz}$ by adjusting the RF modulation power.

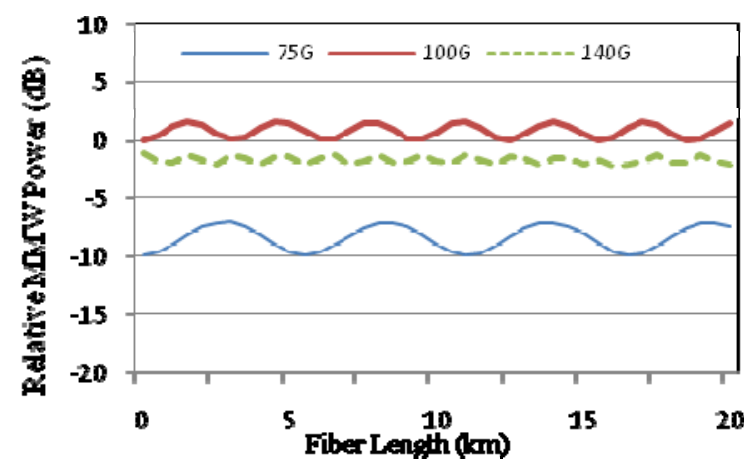

Fig. 7 Chromatic dispersion power penalties vs. fiber length at three indicated MMW frequencies. Fiber attenuation has been deliberately neglected. The power is relative to the power of the $100 \mathrm{GHz}$ signal without fiber link.

\section{CONCLUSION}

We have demonstrated a simple method to generate tunable MMW signals covering the frequency range from $75 \mathrm{GHz}$ to $140 \mathrm{GHz}$. The novel filter configuration removes the need for polarization control and optical path length stabilization, thereby reducing cost and complexity. The generated MMW signal has a low phase noise, governed only by the phase noise of the modulator drive signal. The MMW signal can be distributed by optical fiber with only a small power penalty due to chromatic dispersion.

\section{REFERENCES}

1. G. K. Chang, J. Yu, Z. Jia and J. Yu, "Novel optical-wireless access network architecture for simultaneously providing broadband wireless and wired services," Proceedings of Optical Fiber Communication Conference, Anaheim, California, March, 2006

2. X. J. Meng and J. Menders, "Optical generation of microwave signals using SSB-based frequency-doubling scheme," Electron. Lett., vol. 39, pp. 103-105, 2003

3. T. Yamamoto, H. Takara, and S. Kawanishi, "Generation and transmission of tunable terahertz optical clock," Int. Topical Mtg. Microwave Photonics, T2-2, pp.97-100, 2002

4. J. Yu, Z. Jia, L. Yi, Y. Su, G.-K. Chang, "Optical millimetre-wave generation or up conversion using external modulators," IEEE Photon. Technol. Lett., vol. 18, pp.265-267, 2006

5. P. O. Hedekvist, B. E. Olsson, and A. Wiberg, "Harmonic generation of photonic microwave frequencies utilizing the properties of a phase modulator," Int. Topical Mtg. Microwave Photonics, pp 189-192, 2003

6. G. Qi, J. Yao, J. Seregelyi, S. Paquet, and J. C. Bélisle, "Millimeter-wave carrier generation using an optical phase modulator and an optical notch filter," Proc. SPIE, vol. 5579, pp. 673-679, 2004

7. T. Yamamoto, S. Kawanishi, A. Ueda, and M. Ishiguro, "Low phase noise laser synthesizer with simple configuration adopting phase modulator and fiber Bragg gratings," ALMA memo \#508, 2004

8. P. Shen, N. J. Gomes, P. A. Davies, W. P. Shillue, P. G. Huggard, and B. N. Ellison, "High-purity millimetre-wave photonic local oscillator generation and delivery," Int. Topical Mtg. Microwave Photonics, pp.189-192, 2003

9. H. Suzuki, M. Fujiwara, K. Iwatsuki, A. Hirata, T. Nagatsuma, "Photonic millimetre-wave generator using intensity and phase modulators for $10 \mathrm{Gbit} / \mathrm{s}$ wireless link", Electron. Lett., vol. 41, pp. $355-356,2005$

10. U. Gliese, S. Norskov, and T. N. Nielsen, "Chromatic dispersion in fiber optic microwave and millimeter-wave links," IEEE Trans. Microwave Theory Tech., vol. 44, pp. 1716-1724, 1996 